\title{
Mobilités internationales et ressources en contexte métropolitain : trajectoires centraméricaines à Mexico
}

\section{Laurent Faret}

\section{(2) OpenEdition Journals}

Electronic version

URL: https://journals.openedition.org/echogeo/14915

DOI: 10.4000/echogeo.14915

ISSN: 1963-1197

\section{Publisher}

Pôle de recherche pour l'organisation et la diffusion de l'information géographique (CNRS UMR 8586)

\section{Electronic reference}

Laurent Faret, "Mobilités internationales et ressources en contexte métropolitain : trajectoires centraméricaines à Mexico ", EchoGéo [Online], 39 | 2017, Online since 28 March 2017, connection on 10 August 2021. URL: http://journals.openedition.org/echogeo/14915 ; DOI: https://doi.org/10.4000/ echogeo.14915

This text was automatically generated on 10 August 2021.

EchoGéo est mis à disposition selon les termes de la licence Creative Commons Attribution - Pas d'Utilisation Commerciale - Pas de Modification 4.0 International (CC BY-NC-ND) 


\title{
Mobilités internationales et ressources en contexte métropolitain : trajectoires centraméricaines à Mexico
}

\author{
Laurent Faret
}

1 Dans une perspective classique de l'analyse des dynamiques urbaines, la ville peut être considérée à la fois comme le lieu d'un ensemble de ressources situées (logement, emploi, services...) existant par leur disponibilité relative pour des populations urbaines, et par les processus socioéconomiques et politiques qui contribuent à produire et organiser ces ressources, où interviennent des acteurs multiples aux intérêts non convergents (Pahl, 1975). Ces ressources, exprimées en termes de marchés (marchés de l'emploi, marché immobilier...) ou de potentialités (accès au système de santé, au système éducatif, à des dispositifs d'aide...), sont l'objet de dynamiques de production en permanente évolution. Ces dernières sont peu perméables à une lecture globale si l'on tient compte du fait que la question de l'accessibilité dépend aussi, et de façon irréductible, des caractéristiques des populations urbaines pour lesquelles l'existence de ces ressources est posée et des rapports de pouvoir entre groupes sociaux dans la ville (Duncan, 1976). La question de la distribution et de l'accès aux ressources a ainsi constitué et continue d'être une pierre angulaire des analyses sur le développement urbain et sur la ville en général, notamment lorsque celle-ci est appréhendée comme un système socio-spatial où s'expriment configurations territoriales et rapports de pouvoir autour de ces ressources et leur allocation (Mack et McElrath, 1964 ; ONU-Habitat, 2012).

2 L'existence d'un ensemble d'opportunités potentiellement accessibles caractériserait ainsi l'attractivité d'un espace urbain, en particulier dans ses capacités à drainer des flux de population migrante. Dans ce sens, l'espace urbain, et plus encore métropolitain, offrirait des avantages objectifs, liés à la diversité des différents secteurs du marché de l'emploi, formel et informel, à la grande variété des types et des 
modalités d'accès au logement ou encore à l'éventail des services publics et privés, en matière de santé, d'éducation, de transport... Le risque d'une telle approche existe cependant, celui d'une vision mécaniste de la ville considérée comme un ensemble de lieux dotés de ressources accessibles et disponibles, même si diversement, que des acteurs urbains organiseraient. Une lecture normative ou prescriptive, à portée fonctionnaliste de la construction des dynamiques urbaines peut en découler. Si l'une des dimensions des dynamiques migratoires vers la ville est bien reliée à cette attractivité, il convient de s'interroger sur la nature du rapport entre l'existence de ressources situées et la possibilité pour des populations en provenance de l'extérieur, et en particulier de l'étranger, d'accéder à celles-ci. En particulier, pour ce qui nous intéresse ici, lorsque les populations migrantes en question sont dans des situations de trajectoires contrariées, de changement par rapport aux projets migratoires initiaux ou de faible recours à des réseaux de connaissance dans ces environnements urbains.

Le travail présenté ici a pour objectif de questionner ce rapport dans un cas trop peu étudié encore, celui des populations centraméricaines d'établissement récent à Mexico, à partir de l'observation des formes d'accès à un environnement métropolitain dans un contexte marqué par un fort niveau de mobilité, de vulnérabilité et d'invisibilité. Hors du contexte proprement mexicain, il s'agit de contribuer à éclairer les enjeux contemporains de dynamiques migratoires où les situations de «trajectoires contrariées » sont devenues plus manifestes au gré de la complexification des parcours. Comme on le verra, les flux migratoires en provenance du Honduras, du Salvador et du Guatemala amènent aujourd'hui dans un nombre de cas non négligeable à des trajectoires non "linéaires", au sens où la présence au Mexique ne peut être restreinte à un simple moment (de quelques heures ou jours) dans un espace/temps de trajectoire vers les États-Unis. L'objectif est de proposer une réflexion sur les conditions de l'être en ville pour des populations migrantes dont le transit laisse place à des présences plus durables, et sur le jeu des tensions entre possibilités et contraintes de l'insertion dans un nouvel environnement urbain ${ }^{1}$. Il s'agit aussi de réfléchir aux formes de production d'urbanité par ces populations dans un contexte métropolitain, c'est-à-dire dont les échelles et mécanismes vont au-delà de la ville de dimension moindre pour laquelle on présupposerait une trame socio-spatiale plus homogène. En mobilisant différents niveaux d'échelle, le but est de conduire une observation des pratiques spécifiques de l'espace urbain en lien avec cette dynamique d'accès à des ressources et de s'interroger sur les différentes interactions socio-spatiales que celles-ci génèrent. Dans une démarche systémique, les échelles considérées ici ont été retenues au gré des interactions situées que le fait migratoire fait apparaître. Celles-ci vont du micro-local et des interactions individuelles (lieu d'arrivée dans l'espace urbain, rues et places) aux espaces locaux urbains (quartiers, délégations et municipes) à l'ordre métropolitain et l'espace régional urbain (articulation des trajectoires avec les parcours extra-urbains). On sait combien les présences migrantes, lorsqu'elles s'inscrivent dans la durée, participent à la production des dynamiques urbaines (Guillon, 1995; Tarrius, 1993). Avec la transformation des formes migratoires et le brouillage des catégories entre transit, étape, installation et circulation, les mobilités migratoires amènent aujourd'hui à d'autres lectures des formes du rapport à l'urbain, notamment dans les contextes de circuits transnationaux marqués par des niveaux élevés d'incertitude et/ou de vulnérabilité (Agier, 1999 ; Bensaâd, 2003 ; Alioua, 2007).

Posée de cette façon, la question de l'accès à des ressources des populations migrantes introduit la prise en compte de dynamiques sociales que l'on pourrait qualifier comme 
étant à l'articulation entre l'être en ville et l'être en migration. À la suite des travaux relevant du champ des études migratoires ${ }^{2}$ plus que des études urbaines, la ressource n'est pas que située/territorialisée, elle est aussi ce que les individus mobilisent : accès à l'information, réseaux de connaissances, production de la ressource par mobilisation d'un ensemble de contacts divers liés à la connaissance de l'environnement socioéconomique, production de stratégies d'adaptation et/ou d'invisibilisation par des pratiques adaptées à un contexte rencontré («mimétisme » avec d'autres populations, usage de la langue et dissimulation des accents, formes de présentation de soi, réification des parcours...). Aborder la question de l'articulation de cet ensemble de moyens renvoie à une analyse des différents niveaux de l'interaction dans l'espace urbain, aux formes de ce qu'Isaac Joseph qualifiait de ressources et répertoires disponibles pour le citadin (Joseph, 1998) et la manière avec laquelle ces formes s'ancrent dans un contexte socioculturel en même temps qu'elles témoignent d'effets de temporalités spécifiques (Hernandez, 2015).

5 Face à la difficulté d'observer ces formes d'insertion, la méthodologie de cette recherche appelle quelques commentaires. En premier lieu, la nature même de la présence en ville, caractérisée par des temporalités fluctuantes, une dispersion spatiale relativement importante et des situations de précarité pour une part notable des populations a rendu nécessaire une démarche foncièrement qualitative, en lien avec des éléments de discussion provenant de la littérature existante et certaines sources secondaires. Les observations empiriques mobilisées dans ce texte proviennent d'un travail d'entretiens et d'observation conduit sur trois périodes entre juillet 2015 et juillet 2016. Les entretiens ont été conduits prioritairement auprès de migrants d'installation récente ${ }^{3}$, auxquels s'ajoutent des entretiens avec des responsables des organisations civiles d'appui et des acteurs publics locaux ou nationaux concernés par le thème. En second lieu, les contextes d'insécurité et d'exposition des populations migrantes à diverses formes de violence de la part des acteurs de la criminalité organisée ou de certains agents des autorités locales et migratoires doivent être signalés ici. Parallèlement aux vulnérabilités qu'ils génèrent, ces contextes ont des effets sur la connaissance des processus en cours : des formes de méfiance généralisée et d'invisibilisation des individus sont largement perceptibles, notamment pour les plus vulnérables d'entre eux (familles, femmes seules, mineurs...). Par ailleurs, ces conditions sont à l'origine pour le chercheur d'une accessibilité limitée à une part des espaces de la présence en ville, notamment dans certains secteurs des périphéries extérieures de l'agglomération et dans les espaces interstitiels de la ville, là où le contrôle par les acteurs criminels est significatif. Ces dimensions limitatives, sans doute trop peu signalées aujourd'hui dans les travaux de sciences sociales, sont aussi partie prenante des réalités d'un contexte métropolitain comme celui du Grand mexico ${ }^{4}$, en même temps qu'elles peuvent relativiser en partie la portée des matériaux de terrain sur lesquels s'appuie l'analyse conduite ici.

\section{Ressources urbaines et migration : échelles et processus}

6 Dans l'espace métropolitain des villes latinoaméricaines, les dynamiques de fragmentation et de division sociale de l'espace métropolitain sont, entre autres caractéristiques, le reflet de formes d'accès aux ressources qui ne peuvent que très 
difficilement être lues à une échelle d'ensemble. En termes d'échelles d'analyse, la place des dynamiques de production métropolitaine et de développement des espaces locaux urbains au sein d'un espace régional urbain donné apparait de plus en plus nécessaire pour saisir les formes de réorganisation des mécanismes d'accès à des ressources, que celles-ci soient relatives au logement ou au marché du travail ou relatives à des services de santé et d'éducation par exemple. Les processus de transformation urbaine associés à la globalisation sont habituellement caractérisés, dans un registre souvent autant métaphorique qu'analytique, par les termes de polarisation des revenus, de dualisation du marché du travail et de fragmentation croissante de l'espace et des sociétés urbaines (Duhau, 2008). Sur ces aspects, on signalera à partir de travaux récents que les échelles de la ségrégation se sont resserrées et que les logiques sociales qui sous-tendent ces processus renvoient à des contextes plus complexes: la localisation dans l'espace métropolitain n'est qu'une dimension à côté des processus sociaux et politiques d'insertion reformulés et à l'individualisation des trajectoires d'insertion (Bayón et Saraví, 2013).

7 Dans la perspective développée ici, il en résulte que l'idée d'une appréhension par les populations migrantes d'un environnement et d'une échelle métropolitaines relève souvent plus d'un effet d'analyse que des expériences propres telles que l'observation permet de les caractériser. L'accès à la ville est plus vraisemblablement la résultante d'un double jeu, celui des interactions à l'échelle locale, voire micro-locale, des contextes d'arrivée/insertion; celui des articulations entre ces dynamiques locales et des facteurs structurant de niveau métropolitain. Pour compléter le cadre d'observation, ajoutons que dans le cas des trajectoires migratoires, les modes d'appréhension d'un environnement donné mobilisent également la référence à un espace physico-social migratoire plus large pour les individus, celui des environnements quittés, atteints préalablement ou objets de projets à réaliser.

En parallèle, la littérature sur l'accès aux ressources en ville pour les populations les plus vulnérables économiquement et socialement signale la dégradation des conditions d'insertion et de reproduction sociale, dans un contexte de renforcement d'inégalités et de transformation des politiques sociales et de gestion urbaine (Ziccardi, 2012; Sehtman y Zenteno, 2015). Les situations de crise et le renforcement des effets du modèle néolibéral dans la production des environnements urbains ont conduit à une érosion des réponses domestiques collectives et de la mobilisation des réseaux d'échange et de solidarité, rendant de plus en plus difficiles la mise en œuvre des stratégies adaptatives. Selon les termes de Gonzalez de la Rocha, les ressources de la pauvreté ont fait place à une pauvreté des ressources, dans le sens d'une dégradation des capacités d'adaptation des populations pauvres aux nouvelles dynamiques urbaines d'exclusion, y compris par l'érosion du recours à des activités économiques informelles (Gonzalez de la Rocha, 2001).

9 En parallèle de ces évolutions d'ensemble, on signalera que les modalités d'accès à la ville, quand elles sont considérées à l'échelle des groupes sociaux ou des individus, continuent de s'inscrire plus immédiatement dans une perspective locale. Ces modalités sont à la fois marquées par des trajectoires d'insertion dans le tissu urbain (y compris physique, de points d'étape dans les parcours de mobilité par exemple) et d'expérimentation d'environnements caractérisés par des situations de développement données, des contextes locaux produits par des dynamiques antérieures propres et par les articulations entre ces espaces et le reste d'un environnement urbain plus large. 
L'espace urbain métropolitain peut ainsi être abordé comme agrégation d'une pluralité d'espaces urbains distincts, avec coexistence et articulation différenciées de différents ordres associés à différents types de contextes urbains, produits de l'inclusion/ production d'espaces plus ou moins reliés à l'ordre métropolitain (Duhau et Giglia, 2012 ; Prévôt-Schapira, 2005).

\section{Trajectoires et lieux de la présence centraméricaine à Mexico}

10 La population en provenance du Triangle nord de l'Amérique centrale (Honduras, El Salvador et Guatemala) est majoritairement dans une mobilité de "transit» au Mexique, selon les formes de structuration des systèmes migratoires régionaux construits sur la période 1970-2010 (Faret, 2015). Par nature difficile, la quantification des mobilités migratoires entre l'Amérique centrale et les États-Unis via le territoire mexicain est soumise à débat et des estimations entre 140 et 400000 migrants en transit par an sont souvent retenues (Olayo Mendez et al., 2014) ${ }^{5}$. Si la migration dite de passage est très majoritaire, un nombre croissant de travaux signalent le prolongement des durées de permanence au Mexique (Nájera, 2016; Cobo et Fuerte, 2013), en grande partie liées aux effets de vulnérabilité avérée des migrants face à des situations de violence, d'augmentation de l'insécurité, de discrimination et de criminalisation (Casillas, 2011; Rodríguez, 2014 ; REDODEM, 2015). Incluant des formes de mobilité interrompue, d'installation dans des espaces d'attente et des transformations des projets migratoires initiaux, cette dynamique va aujourd'hui au-delà des régions traditionnelles d'immigration dans le Sud du pays et génère des formes d'installation, de temporaires à durables, en particulier dans les contextes urbains, nœuds des déplacements et lieux de l'accès potentiel à des ressources (Tinoco, 2012).

11 Si les bases historiques de la présence centraméricaine au Mexique renvoient à la période des conflits armés dans l'isthme centraméricain ${ }^{6}$, les différences entre les migrations des années 1975-1990 et la migration actuelle sont notables, à la fois dans les caractéristiques sociodémographiques de la population et les contextes de mise en mobilité. De façon générale, et malgré l'absence de données précises, les flux d'aujourd'hui apparaissent plus divers en termes d'âge, de sexe, de niveau de scolarisation et d'origine géographique des migrants. Leur point commun est la très grande vulnérabilité des personnes en déplacement dans leur lieu d'origine, que celleci soit liée à l'augmentation de la violence, notamment urbaine, au cours des deux dernières décennies ou à la dégradation des conditions d'accès aux marchés du travail de la région (PEN, 2016; García et Villafuerte, 2014). Une autre dimension est la prédominance d'une migration très majoritairement sans documentation migratoire (ou dont le visa ayant permis l'entrée sur le territoire a expiré). Pour la période récente, l'obtention du statut de réfugié ou d'un visa humanitaire ne concerne qu'une partie très restreinte des migrants (Cobo et Fuerte, 2013) ${ }^{7}$. La migration masculine reste majoritaire (Rodríguez, 2014), mais la féminisation des flux est devenue significative, ce que les études de la fin des années 1980 avaient déjà commencé à enregistrer (O’Dogherty, 1989). La migration de mineurs, accompagnés mais surtout non accompagnés, est devenue importante, en particulier de jeunes adolescents (Rodríguez, 2014 ; UNICEF, 2016) et témoigne de l'absence d'alternatives dans les régions d'origine. Le niveau moyen de scolarité est plus bas que celui des périodes des guerres civiles, 
attestant d'un élargissement de la migration à des secteurs plus larges des pays d'origine. Concernant la région urbaine de Mexico, la migration salvadorienne est la plus ancienne, ce qui se traduit par l'existence de réseaux sociaux constitués, même s'ils ne sont que partiellement mobilisables par les populations récemment installées. La migration en provenance du Honduras a connu la croissance la plus significative sur la période récente, dans un environnement d'incertitudes plus élevé encore, pour des migrants très souvent dépourvus d'expérience migratoire antérieure ${ }^{8}$.

De façon générale, la région urbaine de Mexico n'est pas la destination initiale de la migration centraméricaine. Pour une grande majorité des migrants dans l'échantillon d'étude, l'installation à Mexico résulte d'une rupture dans un projet initial orienté vers les États-Unis. Mexico apparaît comme un espace de réorientation des trajectoires, un point entre au moins deux types de flux : une trajectoire interrompue dans le parcours Amérique centrale / États-Unis ou une expulsion des États-Unis sans possibilité de retour dans le pays d'origine. On y arrive donc du sud mais aussi du nord. L'analyse de ces types de trajectoires explique en grande partie les conditions d'insertion dans l'espace métropolitain. Dans le premier cas, les situations rencontrées témoignent de l'insuffisance de ressources économiques et sociales pour prolonger le déplacement vers le Nord. Le coût du déplacement et son augmentation récente du fait de la montée en puissance des réseaux de passeurs ${ }^{9}$, la faible connaissance des routes migratoires et une possibilité très restreinte de s'appuyer sur des réseaux de connaissance en sont les principales caractéristiques. Dans le second cas, Mexico apparaît comme une localisation de repli après une expulsion des États-Unis. Ici, ce sont à la fois les situations de violence vécues ou craintes dans l'environnement d'origine et, de façon moins marquée, les difficultés d'accès au marché du travail dans les pays d'origine qui empêchent le retour. Rester à Mexico apparaît alors comme une alternative, au-delà des difficultés rencontrées, en même temps qu'une possibilité d'entretenir l'espoir d'une nouvelle migration.

13 En lien avec ces trajectoires diversifiées et des parcours souvent complexes, les migrants centraméricains sont spatialement dispersés à l'échelle de l'aire métropolitaine de Mexico. Aucune concentration spatiale d'importance ne peut permettre de parler de quartiers centraméricains à cette échelle, encore moins d'espaces d'un entre-soi ethnico-national. Historiquement, de petits espaces de proximité résidentielle à des échelles locales peuvent exister, notamment à partir du jeu des collectifs d'accueil des réfugiés des années de guerre. Mais la dispersion reste une caractéristique première, en lien souvent avec la question de l'invisibilité, dimension sur laquelle on reviendra. Cet hétérolocalisme de fait (au sens de Zelinsky et Lee, 1998), résulte de trajectoires migratoires extrêmement diversifiées, où les effets de réseaux sociaux ne jouent que pour une part. Les éléments individuels des parcours et les opportunités rencontrées localement au moment de l'arrivée en ville apparaissent plus prégnants pour les migrants d'installation récente, notamment ceux arrivés depuis moins de 2 à 3 ans. Avant de lire les trajectoires aux échelles locales, signalons que les données du recensement de 2010 permettent une appréciation, même si assez imprécise, puisque la part de la population la plus mobile et/ou la plus récemment arrivée est notoirement sous renseignée. Selon cette source indirecte, une spatialisation à l'échelle des délégations (pour la ville de Mexico) et des municipes (pour la partie en conurbation de l'État de Mexico) permet une première caractérisation. Pour les Salvadoriens, les espaces de première périphérie anciennement intégrés sont les lieux d'une présence plus importante qu'ailleurs: 
Tlalpan, Naucalpan et Atizapán notamment. Ici les logiques d'insertion des années 1980 restent vraisemblablement perceptibles, même si moins opératoires aujourd'hui. Pour les Honduriens, la présence plus récente se lit en partie par une surreprésentation dans les municipes du nord de l'agglomération (Tultitlan, Ecatepec de Morelos et Cuautitlán Izcalli notamment), périphéries plus lointaines de l'agglomération, au niveau socioéconomique plus bas et dont l'insertion dans l'espace métropolitain n'est que partielle. Pour la population originaire du Guatemala, une coexistence d'installations en situation centrale (Benito Juarez) et périphérique (Ecatepec) apparait.

Pour autant, on peut établir que la présence centraméricaine à Mexico est une réalité devenue significative selon une autre dynamique spatiale, récente et évolutive, plus lisible à une échelle locale, celle qui résulte des lieux d'interruption des parcours et de recherche d'appui dans l'environnement métropolitain. En ce sens, la géographie de la présence centraméricaine est en grande partie liée aux axes du déplacement, en lien avec la migration dite de transit. Nœud sur les routes migratoires qui conduisent vers les États-Unis, Mexico est le point de la concentration des réseaux routiers et ferroviaires nationaux. Une cartographie des routes migratoires ${ }^{10}$ fait apparaître la région urbaine de Mexico comme le lieu des ruptures de déplacement. Pour les mouvements en bus ${ }^{11}$, les grandes jonctions inter-urbaines aboutissent ou partent des principaux terminaux de la capitale. La distribution des réseaux d'autobus de niveau secondaire est beaucoup plus complexe, mais les polarités que constituent les centralités de second ordre dans l'espace métropolitain sont également marquées, et certaines mobilités migratoires vers le Nord sont en partie assurées par ce réseau secondaire. Le moindre coût du transport et des contrôles migratoires plus rares expliquent des déplacements de courte distance, rendant nécessaire un apprentissage des destinations successives à atteindre. Dans le grand Mexico, cette mobilité par sauts successifs apparaît plus compliquée qu'ailleurs, une connaissance de l'espace métropolitain étant difficile à acquérir ${ }^{12}$. Les déplacements migratoires à travers la grande région urbaine reposent également sur le réseau de transport ferroviaire des marchandises (Casillas, 2008 ; Aragon, 2014). Les convois en provenance d'Ixtepec et de Medias Aguas dans l'isthme de Tehuantepec ou de Veracruz depuis le Golfe du Mexique convergent au nord de Mexico. Ces trains permettent le déplacement des migrants depuis les régions sud du pays vers le centre du territoire national, avant une redistribution plus au nord selon les différentes destinations aux États-Unis ou les points de passage de la frontière (routes de Mexicali, el Paso, Laredo...).

\section{Faire ressource à Mexico}

Dans la logique de l'installation, il s'agit pour les migrants de « faire ressource » dans la ville, au sens de créer un certain nombre de conditions permettant une permanence audelà des moments d'attente avant un prochain déplacement. La différence avec le transit apparaît ici clairement: la pérennité relative des solutions mises en œuvre l'emporte sur la stratégie de mobilité. Aux recherches de moyens et de ressources pour le déplacement se substitue la recherche de formes d'ancrage et de moyens de stabiliser une présence qui se prolonge.

$16 \mathrm{Du}$ point de vue des logiques territoriales, les lieux dans la ville doivent ici être appréhendés à des échelles micro-locales et au regard de leur articulation avec des territoires locaux. On observe alors l'existence de centralités dans les logiques de 
présence et d'installation en ville : les espaces aux abords des voies de transport et les points de transbordement en sont une première dimension. C'est très souvent à partir de ces points, ou dans leur voisinage immédiat, que s'opère pour les arrivants la première mise en relation avec l'environnement local, à la fois par des formes d'interaction avec les acteurs locaux (migrants en transit, population locale et autorités) et par une exploration progressive en direction des tissus urbains proches. De ce point de vue, la place des centralités secondaires dans l'espace métropolitain est relativement significative : places publiques, églises, marchés ou parcs urbains jouent simultanément le rôle d'espaces d'attente/repos ou d'accès à des aides ponctuelles, notamment sous forme de sollicitation d'aide dans la rue. Sur ce dernier point, les axes routiers et les carrefours entre grandes avenues sont des lieux d'occupation fréquents de l'espace public. Ici la tension reste significative entre possibilité d'accéder à des ressources et exposition à des formes de rançonnage ou de violence.

Pour les migrants dépourvus de réseaux de connaissances dans la ville, cas fréquent dans l'échantillon d'enquête, le cheminement permettant une première insertion débute en ces lieux, et les récits d'accès par des contacts aléatoires à un hébergement temporaire ou une première possibilité d'emploi sont les plus fréquents. Une autre polarité significative, en partie en lien avec ce qui précède, est constituée par les lieux d'hébergement temporaire mis en place par les organisations civiles et religieuses locales, à Mexico et dans certains municipes de la conurbation. Circulant au sein des populations en mouvement, l'information sur l'existence de ces structures oriente une partie des trajectoires. Un exemple significatif est celui de la Casa del migrante San Juan Diego de Tultitlan-Lecheria, foyer d'accueil qui a joué jusqu'à sa fermeture en juillet 2012 un rôle d'espace de polarisation important, permettant un hébergement d'appoint pour les migrants en transit. Fermé suite aux récriminations des riverains dénonçant la concentration d'une population stigmatisée et perçue comme dangereuse, le diocèse de Cuautitlan a transféré le foyer à San Bartolo Huehuetoca, soit à $20 \mathrm{~km}$ plus au nord, en lointaine limite de l'espace métropolitain. Si ce lieu n'offrait qu'un hébergement sur la route migratoire, sa notoriété a aussi joué sur la canalisation des populations qui sont restées à Mexico.

18 Une autre forme de polarisation dans la ville est constituée par les structures des organisations civiles d'appui et d'orientation des migrants, dont le registre n'est pas celui de l'hébergement de quelques jours (comme celles signalées précédemment) mais un accompagnement plus durable, juridique et d'aide à l'insertion. Les foyers des organisations telles que Sin Fronteras, Casa Tochan, Cafemin ou Casa de los Amigos sont des lieux qui font centralité pour les migrants accueillis, dans des quartiers plus résidentiels que les lieux de première arrivée, au gré des bâtiments que ces organisation réussissent à utiliser pour leurs activités, souvent dans des extensions anciennes et intégrées de la métropole. Ces lieux sont alors la base d'une pratique de la ville qui va en s'élargissant, avec un usage assez restreint des transports publics au départ mais devenant un peu plus significatif au gré des expériences et des démarches entreprises (démarches administratives, recherche de revenus, premières formes de socialisation hors du foyer, etc...).

Dans les contextes décrits ci-dessus, l'accès aux ressources de l'environnement métropolitain est fortement contraint. Dans la logique de l'installation, les questions de l'accès à un logement, la possibilité de générer des ressources économiques ou l'accès aux services de santé sont des préoccupations permanentes, objet de réajustements 
continus en fonction d'opportunités et de contraintes elles-mêmes évolutives au gré du contexte urbain. En parallèle, la situation vis-à-vis du statut légal de présence effective sur le territoire mexicain conditionne en grande partie l'accès à ces ressources, faisant de la question de l'obtention de documents migratoires une préoccupation diversement prise en charge par les individus en fonction de leurs trajectoires. Par ailleurs, la vulnérabilité expérimentée lors des parcours ayant conduit à Mexico ne prend évidemment pas fin avec l'installation: la question de la sécurité physique et psychologique est présente en filigrane dans l'ensemble des expériences de l'environnement urbain, où les interactions avec la population locale et/ou les autres groupes migrants sont l'objet de négociations permanentes.

Sauf dans de rares cas qui relèvent aujourd'hui de l'exception ${ }^{13}$, l'accès à des ressources économiques relève des activités de type informel, marquées par une forte précarité. Les domaines les plus fréquemment signalés dans l'enquête comme premier accès à l'emploi sont ceux de l'embauche ponctuelle dans des activités localisées à proximité des points d'arrivée des flux : aide à la décharge de camions, travail de manœuvre sur des chantiers de construction, ateliers de réparation des pneus ou de mécanique auto. Avec un peu plus d'ancienneté, travail domestique, auxiliaire de petit commerce ou restaurant, activité de garde de bâtiments ou parkings... Les activités de prostitution ne sont pas absentes non plus. En grande périphérie, le travail d'ouvrier agricole journalier est également signalé. Le plus fréquemment, l'accès à ces activités ne se fait pas par la mobilisation de réseaux de connaissances mais par des démarches de recherche quotidienne pour des activités généralement de courte durée, très faiblement rémunérées. Les logiques d'accès à des emplois plus stables sont ensuite très diversifiées, mais relèvent dans l'ensemble de contacts établis au fur et à mesure de l'expérience urbaine, et de la possibilité de mobiliser les informations réunies au gré des expériences récentes. À la différence d'autres contextes migratoires, les réseaux d'interconnaissance apparaissent comme restreints, en particulier pour constituer une ressource à l'accès à l'emploi : une grande majorité des personnes de l'échantillon indiquent n'avoir pas eu de contact avec un proche ou un parent avant leur arrivée en ville, et l'obtention d'un emploi grâce à des parents déjà présents est une exception. On peut avancer l'hypothèse que cette déficience des réseaux sociaux tient surtout à la nature de la migration centraméricaine, plus éclatée, non orientée vers Mexico, mais aussi sans doute au caractère récent du processus, donc en cours de structuration. Sur la question de l'accès à l'emploi, on signalera également que des secteurs d'activité apparaissent aujourd'hui comme des possibilités d'embauche en développement, en particulier dans l'environnement industriel du nord de la métropole. Pour certaines entreprises de sous-traitance de l'industrie automobile et chimique ou de recyclage des déchets, une demande existe pour une main d'œuvre prête à accepter des conditions de travail très précaires et hors du marché formel de l'emploi. Comme dans d'autres contextes migratoires, l'existence d'un sous-marché de l'emploi très exposé et non rempli par les populations locales semble exister.

Quelle que soit l'origine de l'installation à Mexico, notamment entre provenance du sud ou du nord, les entretiens conduits permettent de dégager des éléments partagés sur le contexte dans lequel se joue cet accès à des ressources. Au sens de travaux antérieurs, la vulnérabilité constatée apparaît comme " un ensemble de situations cumulatives qui ont pour corolaire des vulnérabilités différentes en intensité, dans l'espace et dans le temps et qui se rétroalimentent " (Silva, 2014). Sur ce point, les récits des migrants centraméricains à Mexico témoignent d'une somme de difficultés et de marginalisation 
liées conjointement aux obstacles du premier accès à la ville, aux difficultés expérimentées lors des premiers mois de l'installation tout comme à la rémanence de situations de violence antérieures (trajectoires migratoires et insécurité des contextes d'origine). Dans des cas qui ne sont pas rares, l'insertion à l'environnement urbain se fait alors que les situations de risque connues lors de l'étape migratoire antérieure sont encore bien présentes : abus et extorsions, violences subies ou séquestration, difficultés du déplacement dans des conditions très précaires, parfois avec contraction de dettes vis-à-vis des familles dans le lieu d'origine. Ces éléments de trajectoire contraignent évidemment les possibilités de l'insertion, comme en témoignent les études sur les situations de besoin d'aide psycho-sociale des migrants en transit (Temores et al., 2015).

Ensuite, la connaissance de l'environnement urbain, d'abord très fragmentaire puis en expansion, se fait dans des conditions de méfiance élevée. Aux dangers signalés précédemment s'ajoute une crainte vis-à-vis des autorités ${ }^{14}$ et une certaine méfiance vis-à-vis des autres Centraméricains, notamment depuis que les membres de maras sont également en situation de mobilité internationale vers Mexico. De même, la faiblesse de réseaux sociaux véritablement utilisables au moment de l'arrivée en ville est un élément qui apparait nettement. Lorsqu'il y a connaissance de personnes sur place, l'aide apportée n'est qu'assez rarement économique ou de facilitation de l'accueil. On note par contre qu'une dimension significative des interactions avec les nationaux est relative à l'information sur les situations de risque, sur le degré de sûreté des lieux, voire en termes d'orientation vers les structures d'aide ou de mise en place de démarches administratives.

Un autre élément commun à l'analyse est celui d'une faible maîtrise des temporalités de l'installation: à l'échelle individuelle, plusieurs éléments peuvent devenir déterminants dans le fait de rester ou non, sans qu'aucun d'entre eux ne s'avère central a priori. La durée d'une démarche administrative (notamment la demande de visa humanitaire ou la régularisation migratoire), l'obtention d'un emploi présentant une certaine stabilité sont des facteurs, tout comme le sont aussi la rencontre d'un(e) conjoint(e), la naissance d'un enfant ou le fait de pouvoir faire venir des membres de la famille. Ces temporalités enchâssées ont souvent pour effet un flottement dans les projets à moyen et long terme et, dans certains cas, produisent une connaissance du milieu d'insertion marquée par des phases d'extension mais aussi de repli.

\section{Trajectoires d'insertion et dynamique métropolitaine : Mexico comme une gateway city?}

Les logiques migratoires des Centraméricains à Mexico montrent que les situations rencontrées, plus ou moins temporaires, très souvent difficiles, rendent nécessaire la mobilisation d'un éventail de pratiques et stratégies qui tirent autant de ressources propres aux individus qu'à la mise en valeur des possibilités expérimentées dans le nouvel environnement atteint, donc de ressources situées. D'un côté, les conditions de l'accès à la ville renvoient à des logiques classiques, telles que par exemple celles décrites depuis longtemps par les travaux de l'anthropologie mexicaine, à la suite de Lourdes Arizpe ou Larissa Lomnitz. De l'autre, les formes d'appréhension de la ville et d'insertion à la ville ont fortement changé. Dans un sens, la fragmentation des expériences de l'urbain semble faire écho à la fragmentation métropolitaine. Dans la perspective croisée des trajectoires migratoires et des dynamiques urbaines, ces 
populations migrantes ont à faire face à la fois à des situations de marge sociale dans le pays dit de transit, de relégation dans des espaces en position périphérique dans le contexte métropolitain, en même temps qu'à des micro-marges dans le tissu urbain aux échelles locales. La maîtrise de l'environnement urbain, la connaissance de ces lieux, l'expérimentation des interactions avec les autres populations et acteurs de la ville témoignent de formes spécifiques d'un être en ville. Celui-ci est marqué par le double jeu de trajectoires interrompues, voire d'impasses en ce qui concerne la poursuite du mouvement, avec ses effets de temporalités suspendues, de production d'un espace/ temps contraint ou l'immobilité devient effective. En parallèle, les logiques d'ancrage, de recherche de ressources locales et de construction de trajectoires d'insertion dans la ville sont réelles. Comme ailleurs, elles conduisent à des formes de reproduction/ transmission, générant dans le temps des mécanismes de consolidation qui vont en s'élargissant.

La place de ces populations, dans des contextes locaux souvent déjà tendus du point de vue des ressources, questionne les possibilités d'insertion. Quelle peut être, en ces lieux de la difficile amélioration des conditions de vie d'une large partie des populations pauvres de la métropole, la place des plus pauvres? Les dimensions de la précarité, ici, ne sont pas à entendre seulement en termes économiques. Ce sont celles aussi des carences dans la possible mobilisation de ressources sociales, celles de la vulnérabilité, de l'exposition aux risques de l'illégalité ou de la très insuffisante réponse des autorités nationales ou locales. Plus largement, l'exercice de la citoyenneté urbaine est dans ces conditions extrêmement restreint pour ces populations. L'expression d'un "droit à la ville " passe par des pratiques, par un être là, mais sa formulation en tant que tel n'est aujourd'hui perceptible que dans des cercles très restreints. Qui plus est, lorsqu'elle existe, c'est porté par des organisations civiles ou de défense des droits de l'homme plus que par des groupes migrants organisés.

Pour autant, un autre questionnement est ouvert. Les observations conduites à Mexico montrent que des formes d'insertion sont à l'œuvre, que la métropole peut aussi faire ressource. D'une certaine façon, les dynamiques de cet ancrage contraint témoignent, en creux, des réalités métropolitaines d'aujourd'hui, en particulier dans les environnements urbains des pays dits du Sud. Nous considérons que les trajectoires observées à Mexico rendent compte de la construction d'une destination migratoire en cours de structuration pour les populations en provenance du Triangle Nord centraméricain. À la suite des travaux sur les nouvelles gateway cities, au sens de Price et Benton (2008) ou, dans un contexte urbain d'immigration antérieure, les travaux de Light (2006), au moins trois dimensions sont à signaler. En premier lieu, la segmentation des marchés de l'emploi et la croissance des activités métropolitaines doivent conduire à mieux analyser la production de niches d'emploi. L'activité dans les secteurs du travail domestique (Toledo, 2008) ou dans les sous-secteurs informels de l'industrie en sont des exemples. Ce processus, observé ailleurs depuis longtemps, n'est vraisemblablement pas absent dans le Grand Mexico. La faiblesse des cadres normatifs, la flexibilité des arrangements locaux et les niveaux élevés de contrôle par des opérateurs aux légitimités construites hors du cadre légal jouent dans ce sens. En second lieu, la métropole apparaît aussi comme le lieu d'une possible invisibilité pour les migrants centraméricains. Au-delà des stratégies classiques de l'association avec des migrants internes (par dissimulation de l'accent, par apprentissage des façons d'être de la population locale...), l'immersion dans les dynamiques urbaines se traduit moins par 
des formes de construction de collectifs nationaux que par l'adoption progressive de normes locales, sous des formes qui tranchent avec les dynamiques d'organisation expérimentées ailleurs. Dans le cas des migrants salvadoriens, et même si les effets de temps sont bien sûr ici notables, la prégnance des réseaux sociaux et des organisations communautaires est incomparable avec celle observée aux États-Unis. L'invisibilisation peut aussi être ici stratégie de défense face aux actions des organisations criminelles et autres agents de l'exploitation de la vulnérabilité migratoire, y compris les autorités locales et nationales. Enfin, en lien avec ce second point, c'est au regard des expériences migratoires que l'installation à Mexico peut être lue. Entre difficulté d'accéder aux États-Unis et impossibilité du retour, la métropole est un entre-deux. Par certains côtés, elle semble l'être plus que d'autres régions du Mexique, notamment au sud ou à la frontière nord, où discrimination et exposition aux opérations d'expulsion par les autorités migratoires sont plus marquées. Pour des populations évoluant dans un contexte psychosocial aussi contraint, l'idée que la métropole puisse constituer un environnement plus apaisé apparaît tangible. Malgré les nombreuses contraintes signalées, Mexico peut être un contexte à la fois moins violent que les lieux d'origine et moins exposé que les lieux de la mobilité.

Dans le même temps, la lecture de ces formes d'ancrage en amorce ne peut pas être découplée des perspectives de mobilité à venir, celles-ci apparaissant comme une toile de fond ancrée soit dans des projets migratoires encore effectifs quoique repoussés, soit dans l'éventualité de nouvelles mobilités comme réponse à un établissement devenant irréalisable. Cette récurrence d'une mobilité en perspective est en elle-même vraisemblablement une forme de ressource, mais elle n'apparait pas comme toujours conciliable avec l'ancrage local. À la différence d'autres groupes sociaux pour lesquels la mobilité s'inscrit de plus en plus comme un élément dans un éventail de possible et de " compétences », celle des populations en situation de transit qui dure est davantage l'expression d'une somme de vulnérabilités. Le couplement des processus d'ancrage par défaut et de mobilité latente apparait comme symptomatique des populations exposées à de tels niveaux de vulnérabilité dans les différents environnements qu'elles expérimentent.

\section{BIBLIOGRAPHY}

Agier M., 1999. L'invention de la ville. Banlieues, townships, invasions et favelas. Paris, Éditions des Archives contemporaines, $176 \mathrm{p}$.

Alioua M., 2007. Nouveaux et anciens espaces de circulation internationale au Maroc. Revue des mondes musulmans et de la Méditerranée, $\mathrm{n}^{\circ}$ 119-120, p. 39-58.

Aragon A., 2014. Migrations clandestines d'Amérique centrale vers les États-Unis. Paris, Presses Sorbonne Nouvelle, $268 \mathrm{p}$.

Arizpe L., 1978. Migración, etnicismo y cambio económico. Un estudio sobre migrantes campesinos a la ciudad de México. México, El Colegio de México, 270 p. 
Azaola E.. 2012. La violencia de hoy, las violencias de siempre. Desacatos, $n^{\circ}$ 40, sept-déc.

Bayón M.-C., Saraví G., 2013. The Cultural Dimensions of Urban Fragmentation. Segregation, Sociability, and Inequality in Mexico City. Latin american perspectives, vol. 40, n² 2, p. 35-52.

Bensaâd A., 2003. Agadez, carrefour migratoire sahélo-maghrébin. Revue européenne des migrations Internationales, vol. 19, $\mathrm{n}^{\circ}$ 1, p. 7-28.

Casillas R., 2011. Usos identitarios y culturales en la transmigración por México. Migración y Desarrollo, vol. $9, \mathrm{n}^{\circ} 17$, p. 145-155.

Casillas R., 2008. Las rutas de los centroamericanos por México, un ejercicio de caracterización, actores principales y complejidades. Migración y Desarrollo, n 10, p. 157-174

Cobo S., Fuerte P., 2013. Los refugiados contemporáneos en México. Perfiles sociodemográficos y patrones de participación laboral. Coyuntura demográfica, n 4, p. 63-71.

Duncan S.-S., 1976. Research Directions in Social Geography: Housing Opportunities and Constraints. Transactions of the Institute of British Geographers., vol. 1, $\mathrm{n}^{\circ} 1$, Houses and People in the City, p. 10-19;

Duhau E., 2008. División social del espacio y exclusión social. In Cordera R., Ramírez Kuri P. y Ziccardi A., Pobreza, desigualdad y exclusión social en la ciudad del siglo XXI. México, Siglo XXI-UNAM, p. 199-211.

Duhau E., Giglia A., 2012. Entre la fragmentación y la interdependencia. Reflexiones en torno al orden metropolitano contemporáneo. In Ziccardi A. (coord.), Ciudades del 2010 : entre la sociedad del conocimiento y la desigualdad social, México, UNAM, p. 165-198.

PEN Programa Estado de la Nación en Desarrollo Humano Sostenible, 2016. Quinto Informe Estado de la Región. PEN CONARE, 5ta edición, San José, 452 p.

Faret L., 2015. Notice Mexique. In Simon G. (coord.), Dictionnaire géohistorique des migrations internationales. Paris, Armand Colin.

García Aguilar Ma. del C., Villafuerte Solís D., 2014. Migración, derechos humanos y desarrollo. Aproximaciones desde el sur de México y Centroamérica. Mexico, UCAC - Juan Pablos Ed., 423 p.

González de la Rocha M., 2001. From the Resources of Poverty to the Poverty of Resources? The Erosion of a Survival Model. Latin American Perspectives, vol. 28, n 4, p. 72-100.

Guillon M., 1995. Immigration et centres urbains. Le cas de Paris. In Gallissot R., Moulin B. (éd.), Les quartiers de la ségrégation. Kartala, p. 149-159.

Hernandez Espinoza R., 2015. De identidades, espacios y miradas. Contribuciones para una fenomenologia de la desigualdad social en el espacio urbano. Estudios demograficos y urbanos, vol. $30, \mathrm{n}^{\circ} 1$, p. $77-102$.

Joseph I., 1998. La ville sans qualités. Paris, Éditions de l'Aube, 209 p.

Light I., 2006. Deflecting Immigration: Networks, Markets, and Regulation in Los Angeles. New York, Russell Sage Foundation, $272 \mathrm{p}$.

Lomnitz L., 1975, rééd. 1998. Cómo sobreviven los marginados. México, Siglo XXI Editores, 229 p.

Mack R.-W., McElrath, D.- C., 1964. Urban Social Differentiation and the Allocation of Resources. The Annals of the American Academy of Political and Social Science, vol. 352, p. 25-32.

Ma Mung E., 1999. La dispersion comme ressource. Cultures \& Conflits [En ligne], n 33-34, printemps-été 1999. http://conflits.revues.org/225 ; DOI : 10.4000/conflits.225 
Morokvasic-Muller M., 1999. La mobilité transnationale comme ressource : le cas des migrants de l'Europe de l'Est, Cultures \& Conflits [En ligne], n 33-34, printemps-été 1999. http:// conflits.revues.org/263; DOI : 10.4000/conflits.263

Nájera Aguirre J., 2016. El complejo estudio de la actual migración en tránsito por México: Actores, temáticas y circunstancias. Migraciones Internacionales, vol. 8, nº 3, p. 255-266.

O’Dogherty L., 1989. Centroamericanos en la ciudad de Mexico. Desarraigados y en silencio. Mexico, Academia mexicana de derechos humanos, $57 \mathrm{p}$.

Olayo-Méndez A., Haymes S., Vidal de Haymes M., 2014. Mexican Migration-Corridor Hospitality. Peace Review, vol. 26, n², p. 209-217.

ONU-Habitat, 2012. Estado de las ciudades de América latina y del Caribe : rumbo a una nueva transición. Programa de las Naciones Unidas para los Asentamientos Humanos, 194 p.

Pahl R.-E., 1975. Whose City? Seconde édition, Harmondsworth Penguin, 306 p.

Prévôt-Schapira M.-F., 2005. De l'usage de la fragmentation urbaine en Amérique latine (vue de Buenos Aires). Bulletin de l'Association de géographes français, $n^{\circ}$ 4, p. 483-495.

Price M., Benton-Short L., 2008. Migrants to the metropolis. The rise of immigrant gateway cities. Syracuse University Press, 428 p.

REDODEM, 2015. Migración en tránsito por México: rostro de una crisis humanitaria internacional. Red de documentación de las organizaciones defensoras de migrantes, Rapport annuel, 133 p.

Rodríguez E., 2014. Migración centroamericana en tránsito por México hacia Estados Unidos : Diagnóstico y recomendaciones. México, Instituto Tecnológico Autónomo de México, 45 p.

Sehtman A., Zenteno E. (coord.), 2015. Continuidades, rupturas y emergencias. Las desigualdades urbanas en América Latina. México, UNAM-PUEC, 342 p.

Silva Quiroz Y., 2014. Vulnerabilidad : un concepto para pensar las migraciones internacionales. In Anguiano M.-E., Cruz R., Migraciones internacionales, crisis y vulnerabilidades. Tijuana, COLEF, p. 385-417.

Tarrius A., 1993. Territoires circulatoires et espaces urbains. Différenciation des groupes migrants. Les Annales de la Recherche Urbaines, $\mathrm{n}^{\circ}$ 59-60, p. 50-60.

Temores-Alcántara G., Infante C., Caballero M., Flores-Palacios F. \& Santillanes-Allande N., 2015. Salud mental de migrantes centroamericanos indocumentados en tránsito por la frontera sur de México. Salud Pública de México, vol. 57(3), p. 227-233.

Tinoco Gonzalez I.-A., 2012. Entre exclusiones e inclusiones. Procesos de inserción social de migrantes centroamericanos en el Valle de México. México, FLACSO, Maestría en ciencias sociales, 179 p.

Toledo González M., 2009. Ser empleada domestica e inmigrante : comunidad emergente de peruanas en la ciudad de México. México, CIESAS, Maestría en antropología social, 132 p.

UNICEF, 2016. Broken Dreams. Central American children's dangerous journey to the United States. United Nations Children's Fund, 12 p.

Zelinsky W., Lee. B.-A., 1998. Heterolocalism: An Alternative Model of the Sociospatial Behaviour of Immigrant Ethnic Communities. International Journal of Population Geography, vol. 4, n 4 , p. 281-298.

Ziccardi A. (coord.), 2012. Ciudades del 2010 : entre la sociedad del conocimiento y la desigualdad social. México, UNAM, 1109 p. 


\section{NOTES}

1. Le travail de recherche dont cet article présente une partie des résultats a été conduit dans le cadre de la participation de l'auteur à différents programmes, en particulier ici le Programme RESUM « Ressources urbaines et trajectoires familiales dans le Mexique des années 2010 » (PICS CNRS 2014-2016) coordonné par A. Ribardière, une mission longue durée IRD en avril-mai 2016 et la participation au LMI MESO (IRD).

2. Voir par exemple Ma Mung, 1999 ; Morokvasic-Muller, 1999.

3. Les modes d'entrée en contact avec les populations migrantes ont été diversifiés afin de couvrir au mieux les différentes situations existantes: via les organisations d'appui, via les structures et lieux de sociabilité (restaurants, espaces d'échange et d'organisation sur internet) et dans différents lieux de concentration temporaire des populations migrantes. Au total, l'analyse porte sur 40 entretiens. Pour leur participation à la mise en œuvre du travail de terrain, l'auteur adresse ici ses remerciements à Marina Pantoja García, Gabriela Vargas Flores et María Luisa Buenrostro Loera.

4. Plusieurs municipes de l'État de Mexico en situation de conurbation présentent les taux de criminalité parmi les plus élevés du pays (El Universal, 16/11/2014 ; Expansion, 24/03/2014). La présence d'organisations criminelles comme Los Zetas et La Famila Michoacana opérant dans des activités d'enlèvements, d'extorsion et de narcomenudeo a été partiellement reconnue par les autorités policières (Sexenio Estado de México, 12/03/2012). Voir aussi Azaola, 2012.

5. Les données de 140000 entrées annuelles à la frontière sud publiées par l'Institut National de Migration (INM) et celles de 104000 expulsions annuelles de Centraméricains par les autorités états-uniennes (Najera Aguirre, 2016) donnent aussi une mesure de l'importance du phénomène sur la période récente.

6. En faisant abstraction ici d'une présence plus ancienne remontant aux années 1950, mais beaucoup moins nombreuse et de nature très différente, celle d'étudiants, de professionnels spécialisés ou de leaders politiques.

7. De 2001 à 2011, le Mexique a enregistré 1905 demandes de ressortissants d'Amérique centrale pour la reconnaissance du statut de réfugiés, par ordre d'importance du Honduras, du Salvador et du Guatemala. 296 statuts de réfugiés ont été délivrés, soit $15 \%$ des demandes.

8. Pour une analyse des dynamiques migratoires centraméricaines à Mexico durant la période des conflits armés, on se référa à O'Dogherty, 1989. Du point de vue des nationalités, l'étude indique que $72 \%$ des migrants enquêtés provenaient du Salvador, $20 \%$ du Guatemala et $6 \%$ du Honduras.

9. La plupart des observateurs des dynamiques migratoires centraméricaines s'accordent sur le fait que les flux sont de plus en plus « encadrés " par des groupes organisés illégaux qui tirent un bénéfice économique de ces mobilités, en particulier depuis 2008-2010.

10. Sur les routes de la migration, voir par exemple Casillas 2008.

11. Moins médiatisée mais tout aussi importante, sinon plus, que le déplacement en train (Rodriguez, 2014)

12. Mais loin d'être anodine, notamment dans la partie nord de la région urbaine, où par sauts successifs les migrants quittent la ville en direction de Huehuetoca, Pachuca et Queretaro.

13. Par exemple dans le cas de migrants avec un bon niveau d'études ou pouvant compter sur un solide réseau d'entraide.

14. Le rapport de la REDODEM indique une augmentation significative depuis 2014 des délits commis par des agents des autorités, en particulier de niveau fédéral (REDODEM, 2015). 


\section{ABSTRACTS}

The article aims to document and analyze the forms of mobilization of resources for Central American migrants entering a new metropolitan environment, that of the urban area of Mexico City. In an attempt to shed light on contemporary migration dynamics, where the situation of "frustrated trajectories" have become more evident as the routes become more complex, the article deals with the relationship between mobility trajectories and installation in the metropolitan area, as with the tensions between migratory resources and urban resources. Beyond transit processes, the short-term or more lasting presence of a mobile population from the Central America's Northern Triangle (Salvador, Honduras and Guatemala) questions the forms of anchorage in the city and the socio-spatial relationships of these populations with the different urban and metropolitan dynamics. If the attractiveness of the urban area of Mexico is real, migrant trajectories are also the result of blocked trajectories, either in an attempt to migrate to the North or due to impossible return to the country of origin. Between disrupted migratory projects and weakness of family and social networks, these populations construct adaptive strategies of settlement and socio-economic integration, building despite vulnerability a new horizon anchored locally.

L'article vise à documenter et analyser les formes de mobilisation de ressources pour des migrants centraméricains accédant à un nouvel environnement métropolitain, celui de la région urbaine de Mexico. En cherchant à éclairer les enjeux contemporains de dynamiques migratoires où les situations de «trajectoires contrariées" sont devenues plus manifestes au gré de la complexification des parcours, l'article propose de réinterroger les rapports entre trajectoires de mobilité et installation dans l'espace métropolitain, mettant en tension ressources migratoires et ressources urbaines. Les présences, courtes ou plus durables, d'une population mobile en provenance du Triangle nord centraméricain (Salvador, Honduras et Guatemala) interrogent les formes d'ancrage dans la ville et les rapports socio-spatiaux de ces populations avec les différentes dynamiques urbaines et métropolitaines contemporaines, au-delà du seul transit. Si l'attractivité de la région urbaine de Mexico est réelle, les parcours de migrants sont aussi le résultat de trajectoires bloquées, soit dans une tentative de migration vers le Nord, soit face à des logiques de retour impossible dans le pays d'origine. Entre projets migratoires contrariés et faiblesse de réseaux familiaux et sociaux d'entraide, ces populations construisent des stratégies adaptatives d'installation et d'insertion socioéconomique, fabriquant malgré la vulnérabilité un nouvel horizon ancré localement.

\section{INDEX}

Mots-clés: migration internationale, dynamique métropolitaine, ressource urbaine, transit, Mexico, Centraméricain

Keywords: international migration, metropolitan dynamics, urban resources, transit, Mexico, Central American

Subjects: Sur le Champ - Sur le Terrain 
AUTHOR

\section{LAURENT FARET}

Laurent Faret, faret@univ-paris-diderot.fr est Professeur à l'Université Paris Diderot et membre de l'UMR CESSMA et du LMI MESO. 